\title{
Sixteen Years of Experience with the Treatment of Advanced Colorectal Cancer in Iran; A Report from Three Institutions

Hasan Jalaeikhoo ${ }^{1}$, Ahmad Khajeh-Mehrizi ${ }^{2}$, Mohammad Zokaasadi ${ }^{3}$, Mohsen Rajaeinejad ${ }^{4}$, Seied Asadollah Mousavi ${ }^{1}$, Mohammad Vaezi ${ }^{1}$, Hosein Kamranzadeh Fumani ${ }^{5}$, Manoutchehr Keyhani ${ }^{6}$, Kamran Alimoghaddam ${ }^{7, *}$, Ardeshir Ghavamzadeh ${ }^{7}$

1. Associate Professor, Department of Hematology and Oncology, AJA Cancer Epidemiology Research and Treatment Center (AJA- CERTC), AJA University of Medical Sciences

2. Department of Hematology and Oncology, Hematology, Oncology and Stem cell Transplantation Research Center, Tehran University of Medical Sciences, Tehran, Iran

3. Department of Hematology and Oncology, AJA Cancer Epidemiology Research and Treatment Center (AJA- CERTC), AJA University of Medical Sciences

4. Assistant Professor, Department of Hematology and Oncology, AJA Cancer Epidemiology Research and Treatment Center (AJA- CERTC), AJA University of Medical Sciences

5. Assistant Professor, Department of Hematology and Oncology, Hematology, Oncology, and Stem cell Transplantation Research Center, Tehran University of Medical Sciences, Tehran, Iran

6. Professor, Department of Hematology and Oncology, Hematology and Oncology Research Center, Vali-Asr Hospital, Tehran University of Medical Sciences, Tehran, Iran

7. Professor, research institute for hematology oncology and stem cell transplantation, Shariati hospital, Tehran University of medical sciences, Tehran, Iran

\section{* Corresponding Author:}

Kamran Alimoghaddam, MD

North Karegar Avenue, Shariati Hospital, Hematology, Research Institute for Hematology Oncology and Stem Cell Transplantation, Teharn Uiversity of Medical Sciences, Tehran, Iran

Telefax: +982188004140

Email: alimgh@ams.ac.ir

Received: 03 Mar. 2018

Accepted: 24 May 2018

\section{ABSTRACT}

\section{BACKGROUND}

Colorectal cancer (CRC) is one of the most common cancers worldwide. Recently treatments of advanced CRC have been immensely improved. In this study we reported the current state of advanced CRC in Iran regarding treatment and outcomes from 2000 to 2016.

\section{METHODS}

370 subjects with stage III or IV of the disease were included in this study. Pathological subtypes other than adenocarcinoma were excluded. Demographics and other relevant clinical data were collected.

\section{RESULTS}

Mean age at diagnosis was $55.4 \pm 12.6$ years. Significant differences regarding the age, sex, primary tumor complication and location, lymph node involvement, and tumor size were not detected between patients with stage III and IV. Overall survival rate at 5 years was $69.5 \%$ (95\% confidence interval: $60.8 \%-76.6 \%)$ and $21.73 \%$ (95\% CI: $12.46 \%-32.70 \%$ ) for patients with stage III and IV, respectively. Analysis of prognostic factors revealed that tumor grade was an independent factor predicting poorer outcome (poorly differentiated vs. well or moderately differentiated). Furthermore, in stage IV of the disease, IVb subgroup was found to be associated with a poorer outcome compared with stage IVa.

\section{CONCLUSION}

Even with the acceptable survival rates and more effective treatments, it seems that clinicopathological characteristics have yet the most important prognostic effect in advanced CRC.

\section{KEYWORDS}

Colorectal neoplasms, Neoplasm metastasis, Survival analysis, Iran

\section{Please cite this paper as:}

Jalaeikhoo H, Khajeh-Mehrizi A, Zokaasadi M, Rajaeinejad M, Mousavi SA, Vaezi M, Kamranzadeh Fumani H, Keyhani M, Alimoghaddam K, Ghavamzadeh A. Sixteen Years of Experience with the Treatment of Advanced Colorectal Cancer in Iran; A Report from Three Institutions. Middle East J Dig Dis 2018;10:160-168. doi: 10.15171/mejdd.2018.105.

\section{INTRODUCTION}

Colorectal cancer (CRC) is the third common cancer and also the third leading cause of cancer related deaths in both sexes in the United States. ${ }^{1}$ There are marked geographical variations worldwide and in some areas such as Asia, the incidences are increasing dramatically within the recent few decades. ${ }^{2}$ The disease is also a noticeable health problem in Iran. The age standardized incidence rates in Iran were estimated to be 8.16 (95\% confidence interval (CI): 6.64 to 9.68) and 6.17 (95\% CI: 5.01 to 7.32) per100000 for men and women, respectively. The incidences have lately shown an increasing pattern because of multiple factors. ${ }^{3}$ In the United States about $20 \%$ patients have distant metastatic disease at the 
time of diagnosis, ${ }^{4}$ which the percent seems to be more in developing countries because of delays in screening or diagnosis. Therefore patients with advanced CRC make a considerable part of patients with CRC. Early chemotherapeutic protocols for advanced CRC mainly included 5 -flourouracil (5-FU) in combination with folinic acid or levamisole. ${ }^{5,6}$ Since the approval of more potent agents such as oxiliplatin and irinotecan as the first line treatment protocols has been vastly changed. ${ }^{78}$ In the recent years, the introduction of monoclonal antibodies such as bevacizumab, resulted in better survival rates in advanced CRC. ${ }^{9}$ Although some results were not in favor of targeted therapies for epidermal growth factor receptor or EGFR (i.e. cetuximab),${ }^{10}$ studies are running in this field. In this study we reported a 16-year survey of treatment and outcomes in patients with advanced CRC (either lymph node positive or metastatic) in three referral centers in Tehran, Iran.

\section{MATERIALS AND METHODS}

\section{Cohort criteria and ethical aspects}

Patients in oncology clinics of three hospitals with documented diagnosis of advanced CRC from April 2000 to August 2016 were included in the study. The study was conducted from February 2016 to May 2017 in Tehran, Iran. Exclusion criteria were non-adenocarcinoma types, stages I or II of the disease, concurrent presence of other serious comorbidities affecting life expectancy, and patients who refused palliative or curative therapy. As non-adenocarcinoma types are a minority of CRCs (more than $90 \%$ of CRCs are adenocarcinoma), they were excluded, so a reasonable survival analysis was practicable. The relevant variables including demographics, primary tumor complication, $\mathrm{T}$ category, size, $\mathrm{N}$ category, grade of tumor, presence of distant metastasis, number of dissected and involved lymph nodes, status of surgical margin, and details of chemotherapy were extracted from the patients' medical records. Chronology of the disease was recorded as dates of diagnosis, start of chemotherapy, relapse, and death (in cases with relapse and death). The patients were followed up until death or relapse occurred or up to the time of analysis, which was March 2017. Advanced CRC was defined as the presence of lymph node involvement (stage III), or distant metastasis (stage IV) at the time of diagnosis. Subgroups of stage IV were categorized as the following: stage IVa was applied to a patient whenever distant metastasis was limited to one single organ (but peritoneum), and stage $\mathrm{IVb}$ was defined as metastases in multiple organs or involvement of the peritoneum.

The study protocol was approved by the Ethics Committees of AJA(Artesh Jomhory Islami IRAN University) and Tehran Universities of Medical Sciences. Informed consent was obtained from all the patients in order to use their medical records' information as a resource for medical research. The authors declare that there is no conflict of interest to disclose.

\section{Treatment protocols}

Treatment protocols for the patients were as follows: single agent chemotherapy with 5-FU and folinic acid or its prodrug (capecitabine), combinations of 5-FU, folinic acid, and oxiliplatin (FOLFOX), combinations of 5-FU, folinic acid, and irinotecan (FOLFIRI), combination regimens including both oxiliplatin, and irinotecan, and other regimens. Some patients received monoclonal antibodies based on the results of genetic tests and socioeconomic status. There were three groups in our study: Bevacizumab group, cetuximab group, and a group who did not receive monoclonal antibodies.

\section{Analyses}

Categorical variables were described as frequencies and percentages and continuous variables were described as means and standard deviations. The survival estimates (both overall and disease-free) were calculated based on Kaplan-Meier method. Median survival times were projected using reverse Kaplan-Meier method and in patients with metastasis only overall survival (OS) rates were assessed. Survival curves were compared using logrank test and the effect of possible prognostic factors on outcome were analyzed by cox proportional hazards model. Any variable with a P value of less than 0.2 were considered valid to enter multivariate analysis. Overall survival was defined as the time from diagnosis to death from any cause. Disease-free survival (DFS) was demarcated as the time from diagnosis to death or relapse of the disease. $\mathrm{P}$ values less than 0.05 were considered as statistically significant. $R$ software version 3.3.2 for windows was used to analyze the data. 
Table 1: Basic characteristics of the studied cohort

\begin{tabular}{|c|c|c|c|c|}
\hline Variable & Category & Stage III & Stage IV & $P$ value \\
\hline Age & Mean \pm SD (years) & $55.60 \pm 12.69$ & $55.07 \pm 12.47$ & 0.70 \\
\hline \multirow[t]{2}{*}{ Sex } & Male & $48.03 \%(n=110)$ & $46.81 \%(n=66)$ & 0.83 \\
\hline & Female & $51.97 \%(\mathrm{n}=119)$ & $53.19 \%(\mathrm{n}=75)$ & \\
\hline \multirow{5}{*}{$\begin{array}{l}\text { Primary tumor } \\
\text { complication }\end{array}$} & Obstruction/perforation & $17.90 \%(n=41)$ & $20.57 \%(\mathrm{n}=29)$ & 0.19 \\
\hline & Hemorrhage & $19.65 \%(n=45)$ & $17.02 \%(n=24)$ & \\
\hline & Anemia & $0.87 \%(\mathrm{n}=2)$ & $2.84 \%(n=4)$ & \\
\hline & Abdominal pain & $4.37 \%(n=10)$ & $9.22 \%(n=13)$ & \\
\hline & None & $57.21 \%(\mathrm{n}=131)$ & $50.35 \%(\mathrm{n}=71)$ & \\
\hline \multirow[t]{4}{*}{ Tumor location } & Ascending colon & $25.76 \%(\mathrm{n}=59)$ & $31.21 \%(n=44)$ & 0.58 \\
\hline & Transverse colon & $5.68 \%(n=13)$ & $7.09 \%(n=10)$ & \\
\hline & Descending colon \& sigmoid & $47.60 \%(n=109)$ & $38.30 \%(\mathrm{n}=54)$ & \\
\hline & Recto-sigmoid \& rectum & $20.96 \%(n=48)$ & $23.40 \%(n=33)$ & \\
\hline \multirow[t]{3}{*}{ Mucin production } & Mucinous & $15.28 \%(\mathrm{n}=35)$ & $12.77 \%(\mathrm{n}=18)$ & 0.58 \\
\hline & Non-mucinous & $13.54 \%(n=31)$ & $11.35 \%(n=16)$ & \\
\hline & Unknown & $71.18 \%(n=163)$ & $75.88 \%(n=107)$ & \\
\hline \multirow[t]{2}{*}{ Lymph nodes } & Mean dissected $\pm \mathrm{SD}$ & $9.83 \pm 5.50$ & $9.12 \pm 5.34$ & 0.30 \\
\hline & Mean involved $\pm \mathrm{SD}$ & $3.21 \pm 2.67$ & $3.14 \pm 4.03$ & 0.87 \\
\hline Tumor size & Mean \pm SD (cm) & $4.95 \pm 2.31$ & $5.51 \pm 2.44$ & 0.08 \\
\hline \multirow[t]{4}{*}{ Grade } & Well differentiated & $37.12 \%(\mathrm{n}=85)$ & $19.86 \%(\mathrm{n}=28)$ & 0.04 \\
\hline & Moderately differentiated & $36.68 \%(\mathrm{n}=84)$ & $37.59 \%(\mathrm{n}=53)$ & \\
\hline & Poor differentiated & $5.68 \%(n=13)$ & $8.51 \%(n=12)$ & \\
\hline & Unknown & $20.52 \%(n=47)$ & $34.04 \%(n=48)$ & \\
\hline \multirow{5}{*}{$\begin{array}{l}\text { Adjuvant } \\
\text { chemotherapy }\end{array}$} & Single-agent & $13.1 \%(n=30)$ & $7.09 \%(\mathrm{n}=10)$ & $<0.001$ \\
\hline & Oxiliplatin -based & $70.31 \%(n=161)$ & $62.41 \%(\mathrm{n}=88)$ & \\
\hline & Irinotecan-based & $5.67 \%(\mathrm{n}=13)$ & $11.35 \%(\mathrm{n}=16)$ & \\
\hline & Combination & $0 \%(\mathrm{n}=0)$ & $11.35 \%(\mathrm{n}=16)$ & \\
\hline & Other & $10.92 \%(\mathrm{n}=25)$ & $7.80 \%(\mathrm{n}=11)$ & \\
\hline \multirow{3}{*}{$\begin{array}{l}\text { Monoclonal } \\
\text { antibodies }\end{array}$} & Bevacizumab & $0.87 \%(\mathrm{n}=2)$ & $25.53 \%(n=36)$ & $<0.001$ \\
\hline & Cetuximab & $0.87 \%(\mathrm{n}=2)$ & $15.60 \%(\mathrm{n}=22)$ & \\
\hline & none & $98.26 \%(n=225)$ & $58.87 \%(\mathrm{n}=83)$ & \\
\hline \multirow[t]{2}{*}{ Survival status } & Alive & $77.73 \%(\mathrm{n}=178)$ & $44.0 \%(n=62)$ & $<0.001$ \\
\hline & Dead & $22.27 \%(\mathrm{n}=51)$ & $56.0 \%(\mathrm{n}=79)$ & \\
\hline Relapse & Relapsed & $36.68 \%(\mathrm{n}=84)$ & N/A & N/A \\
\hline
\end{tabular}

\section{RESULTS}

\section{Cohort characteristics}

Of 689 patients with CRC a total number of 370 eligible patients were included in this study. Mean age at the time of diagnosis was $55.4 \pm 12.6$ years. Male and female patients were $47.6 \%(n=176)$, and $52.4 \%(n=194)$, respectively. 229 patients $(61.9 \%)$ had stage III, and the remaining 141 had stage IV (38.1\%) of the disease. Among the 168 symptomatic patients $(45.4 \%$ of all patients) the most common symptoms were hemorrhage, and bowel obstruction, each accounting for 69 patients (41.1\% of symptomatic patients). There were no significant differences between the patients with stage III and IV regarding the age, sex, primary tumor complication and location, lymph node involvement, and tumor size. The only difference observed between the two groups was tumor grade (more well differentiated tumors in patients with stage III, $p=0.04)$ and presence of metastasis $(p<0.001)$. Basic characteristics of the studied cohort based on stage are demonstrated in table 1. 


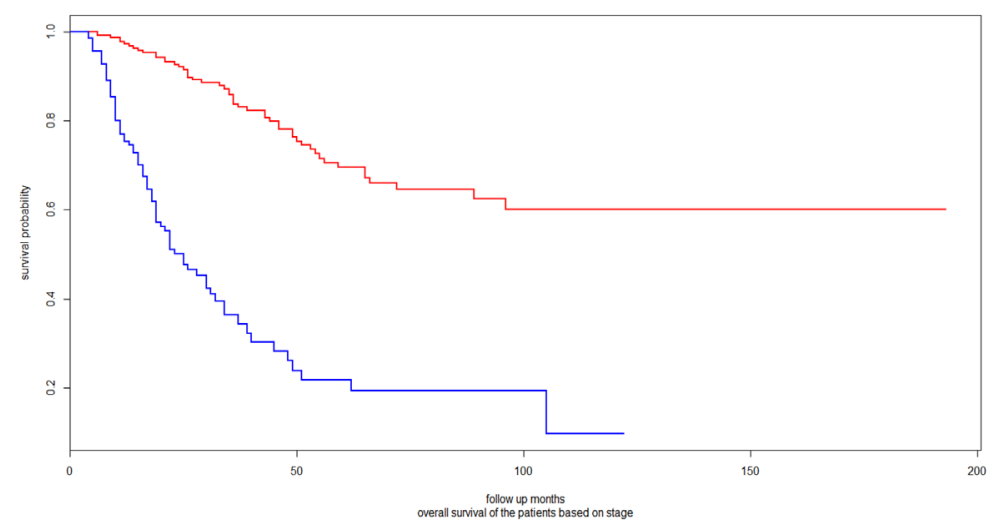

Fig.1: Overall survival of the patients is depicted based on stage. Red curve reflects stage III and blue curve is for stage IV.
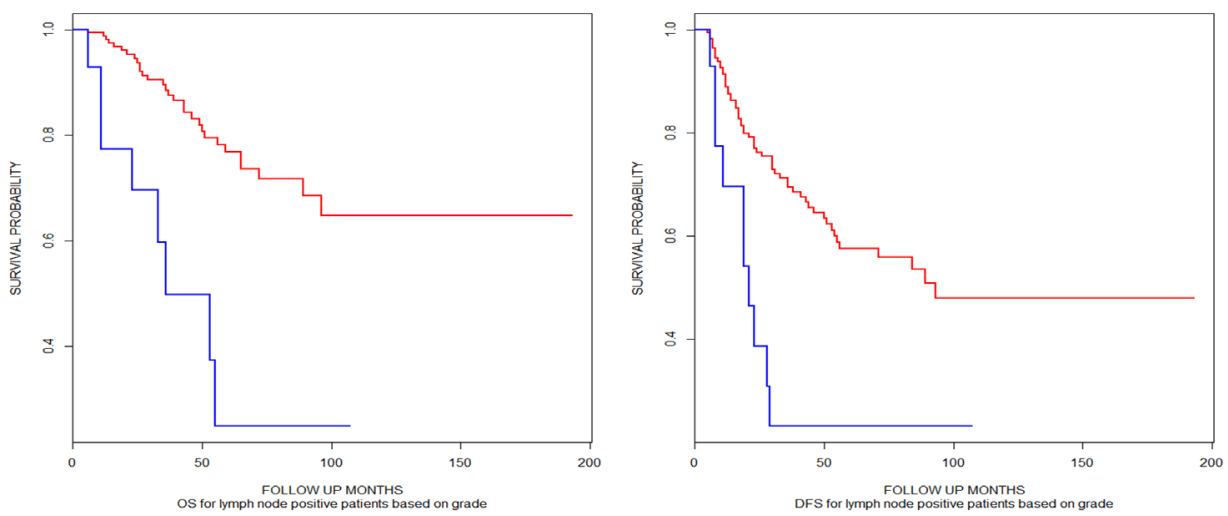

Fig.2: Overall and disease free survival curves of patients with stage III based on grade is shown. Red curve is for moderate or well differentiated and blue curve is for poorly differentiated tumors.

\section{Survival analysis}

Median follow-up time was 72 months. Survival analysis among all patients using Kaplan-Meier method revealed 5- and 10-year overall survival rates of 53.2\% (95\% CI: $46.3 \%-59.7 \%$ ) and $43.7 \%$ (95\% CI: $34.9 \%$ - 52.2\%), respectively. The probability of survival was significantly higher for patients with stage III rather than those with metastasis. 5-year overall survival (OS) rates for stages III and IV were $69.5 \%$ (95\% CI: 60.8\% - 76.6\%) and $21.73 \%$ (95\% CI: $12.46 \%-32.70 \%$ ), respectively $(p<0.001$, figure 1$)$.

Among the patients with stage III CRC, the OS and DFS rates were significantly different for poorly differentiated tumors compared with moderate or well differentiated ones. While 5-year OS and DFS rates for poorly differentiated tumors were $24.9 \%$ (95\% CI: $4.22 \%-54.2 \%$ ) and
23.2\% (95\% CI: $5.62 \%$ - 47.75\%), respectively, these rates were $76.7 \%$ (95\% CI: $67.1 \%-73.9 \%)$ and $57.5 \%$ (95\% CI: $47.9 \%-66.0 \%)$ for non-poorly differentiated tumors ( $p<0.001$ for OS and $p=0.002$ for DFS, figure 2).

Moreover analyses of the data of the patients with stage III did not show any significant differences in subgroups categorized by T classification ( $p=0.97$ and 0.84 for OS and DFS, respectively), $\mathrm{N}$ classification ( $p=0.79$ and 0.35 for OS and DFS, respectively), tumor location ( $p=0.25$ and 0.90 for OS and DFS, respectively), type of adjuvant therapy ( $p=0.46$ and 0.59 for OS and DFS, respectively), production of mucin ( $p=0.35$ and 0.15 for OS and DFS, respectively) and primary tumor complication (symptomatic vs. asymptomatic patients; $p=0.70$ and 0.38 for OS and DFS, respectively). Results of log-rank tests for analyzed variables are summarized in table 2 . 
Table 2: Differences in overall survival for all patients in different categories

\begin{tabular}{|c|c|c|c|c|}
\hline Variable & Category & Median OS time (months) & $\begin{array}{c}\text { 2-year overall survival rate } \\
(95 \% \mathrm{CI})\end{array}$ & $P$ value \\
\hline \multirow[t]{2}{*}{ Sex } & Male & 96 & $76.2 \%(68.5 \%-82.3 \%)$ & \multirow[t]{2}{*}{0.618} \\
\hline & Female & 65 & $76.9 \%(69.8 \%-82.5 \%)$ & \\
\hline \multirow{2}{*}{$\begin{array}{l}\text { Primary } \\
\text { complication }\end{array}$} & Presented with complication & 65 & $76.1 \%(68.9 \%-81.8 \%)$ & \multirow[t]{2}{*}{0.882} \\
\hline & Without complication & 72 & $77.0 \%(69.3 \%-83.1 \%)$ & \\
\hline \multirow[t]{6}{*}{ Tumor location } & Ascending colon & N/A & $79.0 \%(68.6 \%-86.3 \%)$ & \multirow[t]{6}{*}{0.807} \\
\hline & Transverse colon & N/A & $81.4 \%(52.4 \%-93.6 \%)$ & \\
\hline & Descending colon & 65 & $78.3 \%(68.6 \%-85.3 \%)$ & \\
\hline & Sigmoid & N/A & $77.1 \%(62.3 \%-86.7 \%)$ & \\
\hline & Recto-sigmoid & 49 & $72.0 \%(45.0 \%-87.4 \%)$ & \\
\hline & Rectum & 96 & $76.0 \%(61.4 \%-85.7 \%)$ & \\
\hline \multirow[t]{2}{*}{ Mucin production } & Mucinous & 65 & $83.6 \%(69.8 \%-91.5 \%)$ & \multirow[t]{2}{*}{0.538} \\
\hline & Non-mucinous & 89 & $84.0 \%(67.8 \%-92.5 \%)$ & \\
\hline \multirow[t]{2}{*}{ Stage } & III & N/A & $92.0 \%(87.3 \%-95.0 \%)$ & \multirow[t]{2}{*}{$<0.001$} \\
\hline & IV & 25 & $50.04 \%(40.52 \%-58.8 \%)$ & \\
\hline \multirow[t]{2}{*}{ Grade } & Well or moderately differentiated & $\mathrm{N} / \mathrm{A}$ & $83.1 \%(77.3 \%-87.5 \%)$ & \multirow[t]{2}{*}{$<0.001$} \\
\hline & Poorly differentiated & 33 & $50.0 \%(28.83 \%-68.0 \%)$ & \\
\hline \multirow{5}{*}{$\begin{array}{l}\text { Adjuvant } \\
\text { chemotherapy }\end{array}$} & Single-agent & N/A & $87.7 \%(70.3 \%-95.3 \%)$ & \multirow[t]{5}{*}{0.268} \\
\hline & Oxiliplatin-based & 96 & $76.6 \%(70.4 \%-81.7 \%)$ & \\
\hline & Irinotecan-based & N/A & $58.3 \%(37.2 \%-74.5 \%)$ & \\
\hline & Combination & 34 & $83.9 \%(49.4 \%-95.7 \%)$ & \\
\hline & Others & 44 & $68.2 \%(44.17 \%-83.6 \%)$ & \\
\hline
\end{tabular}

Median OS time for patients with stage IV was 25 months. In this subgroup a clinically significant but statistically borderline difference was found between the patients with single or multiple metastases. The 5-year OS rate was $24.4 \%$ (95\% CI: $13.79 \%-36.6 \%$ ) for patients with single organ metastasis compared with zero for patients with peritoneal or multiple organ involvements $(p=0.06)$. No significant differences were observed for grade $(p=0.13)$, tumor location $(p=0.46)$, addition of monoclonal antibodies to treatment $(p=0.15$ ), primary tumor complication (symptomatic vs. asymptomatic patients; $p=0.96)$, type of adjuvant chemotherapy $(p=0.23)$, and mucin production $(p=0.54)$

\section{COX proportional hazards model}

In all patients, metastatic disease and poorly differentiated tumors were independent prognostic factors of poorer OS after adjusting for age, type of adjuvant treatments, and $\mathrm{T}$ classification $(\mathrm{HR}=5.20 ; 95 \% \mathrm{CI}$ :
$3.29-8.20, p<0.001$ and $2.68 ; 95 \%$ CI: $1.47-4.90, p$ $=0.001$ for metastasis and poorly differentiated tumors, respectively). In patients with stage III after performing univariate analysis on age, sex, tumor grade, primary complication (symptomatic vs. asymptomatic), lymph node involvement ( $\mathrm{N}$ classification), tumor location, and adjuvant chemotherapy type, the only independent factor affecting OS (adjusted for age) and DFS was tumor grade. In multivariate analysis hazard ratio (HR) of poorly differentiated tumors was 3.88 (95\% CI: $1.77-8.49$; $p=0.003)$ and $2.87(95 \% \mathrm{CI}: 1.46-5.63 ; p=0.002)$ for OS and DFS, respectively. In metastatic CRC after univariate analysis and adjusting for type of adjuvant chemotherapy, age at the time of diagnosis, addition of monoclonal antibodies to treatment, and $\mathrm{T}$ classification, independent predictors of inferior outcome were found to be poorly differentiated tumors and stage IVb (peritoneal or multiple organ involvement). HRs calculated by multivariate analysis were 3.22 (95\% CI: 1.73 - 5.99; 
Table 3: Results of univariate and multivariate analysis

\begin{tabular}{llccc}
\hline Covariate & $\begin{array}{c}\text { Unadjusted } \\
\text { (Hazard Ratio) } \mathbf{( 9 5 \%} \mathbf{C I})\end{array}$ & $\boldsymbol{P}$ value & $\begin{array}{c}\text { Adjusted HR } \\
\mathbf{( 9 5 \%} \mathbf{C I})\end{array}$ & $\boldsymbol{P \text { value }}$ \\
\hline All patients & & & & \\
\hline Stage (IV/III) & $5.39(3.75-7.77)$ & $<0.001$ & $5.20(3.29-8.20)$ & $<0.001$ \\
\hline Grade (poorly differentiated/non-poorly diff) & $3.07(1.78-5.29)$ & $<0.001$ & $2.68(1.47-4.90)$ & 0.001 \\
\hline Stage III overall survival & & & & \\
\hline Grade (poorly diff/non-poorly diff) & $4.02(1.84-8.79)$ & $<0.001$ & $3.88(1.77-8.49)$ & 0.003 \\
\hline Stage III disease-free survival & & & & 0.002 \\
\hline Grade (poorly diff/non-poorly diff) & $2.87(1.46-5.63)$ & 0.002 & $2.87(1.46-5.63)$ & \\
\hline Stage IV overall survival & & & & \\
\hline Grade (poorly diff/non-poorly diff) & $3.07(1.78-5.29)$ & $<0.001$ & $3.22(1.73-5.99)$ & $<0.001$ \\
\hline Stage subgroup (IVb/IVa) & $1.19(1.14-1.24)$ & $<0.001$ & $1.20(1.13-1.28)$ & $<0.001$ \\
\hline
\end{tabular}

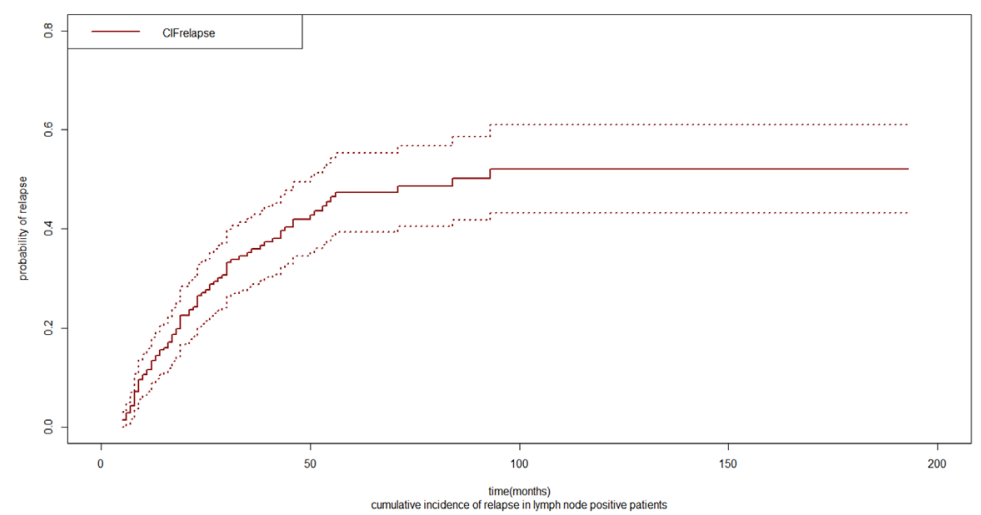

Fig.3: Cumulative incidence of relapse in patients with stage III is demonstrated with $\mathbf{9 5 \%}$ confidence intervals.

$\mathrm{p}<0.001)$ and 1.20 (95\% CI: $1.13-1.28 ; \mathrm{p}<0.001)$ for grade and stage $\mathrm{IVb}$, respectively. Results of multivariate analysis are shown in table 3 .

\section{Relapse incidence}

For patients with stage III the cumulative incidence of relapse at 5 and 10 years were $43.66 \%$ (95\% CI: $36.41 \%-51.67 \%)$ and $52.08 \%$ (95\% CI: $53.51 \%$ $61.24 \%$ ), respectively (Figure 3 ).

\section{DISCUSSION}

In this study we aimed to assess the current state of advanced CRC in Iran. Unlike stage II of the disease, advanced CRC has been proven to be benefited from treatment in previous studies. ${ }^{11}$ It has been demonstrated in earlier studies that palliative chemotherapy in metastatic CRC offers a significant superior survival contrary to best supportive care (BSC). ${ }^{12}$ Therefore BSC is not considered as a proper alternative anymore. The treatment of advanced CRC has been changed significantly during current decades. Early chemotherapeutic protocols like what presented in the study of De Gramont and colleagues in 1980's or study of Laurie and co-workers were mainly consisted of 5-FU based regimens. ${ }^{6}, 13$ More recently approval oxiliplatin, for the treatment of advanced CRC has improved the outcomes of the treatment. ${ }^{14-16}$ Also addition of irinotecan, an inhibitor of topoisomerase I, to 5-FU based regimens have been resulted in better treatment outcomes. $^{7}$

In this study the 5-year OS rates of patients with stage III was $69.5 \%$ (95\% CI: $60.8 \%-76.6 \%$ ), which is comparable to previous studies. Former reports such as the study of Glimelius and others have shown the 5-year OS of $48 \% \pm 3 \%$ to $55 \% \pm 3 \%$, and $48 \pm 4 \%$ to $51 \% \pm 4 \%$ 
in patients with stage III of colon and rectum cancers, respectively. ${ }^{11}$ More recent studies reported better outcomes with stage III of colon cancer. Results of MOSAIC study conducted by Andre and colleagues revealed 6-year OS of $68.7 \%$ to $72.9 \%$ based on treatment regimens. ${ }^{15}$

We also reported a median OS time of 25 months in patients with metastasis. This finding is slightly better than previous studies. The study of Saltz revealed a median OS time of 19.9 to 21.3 months in patients with metastasis. ${ }^{9}$ Similarly the study of Diaz-Rubio and colleagues on 480 patients with metastasis showed median OS time of 20.0 to 23.2 months in different treatment groups. ${ }^{17}$ Results of phase III MRC COIN trial on 1630 patients with advanced CRC showed lesser median OS times in intention to treat population (14.4 to 15.8 months). ${ }^{18}$ Furthermore patients joined OPTIMOX1 study, which was a randomized clinical trial, reached to a median OS time of 19.3 to 21.2 months in two different arms of the study. ${ }^{19}$ In the same way, results from NO16966 study revealed median OS time of 17.7 to 21.6 months in the whole cohort. ${ }^{20}$

As expected we detected higher stage as an independent prognostic factor of poorer outcome. We also found out higher pathological grade was independently linked to more deaths. This finding was formerly noticed in the literature like study of Gill and co-workers. ${ }^{21}$ In that study the HR of high grade versus low grade was 1.34 (95\% CI: $1.12-1.60, p=0.0017)$; however in our study the HR was more prominent $(\mathrm{HR}=2.68,95 \% \mathrm{CI}$ : $1.47-4.90 ; p=0.001)$. We figured out that in patients with metastatic CRC, the stage IVb subgroup (peritoneal or multi-organ involvement) had $20 \%$ increased probability of death. Results from the trials of Analysis and Research in Cancers of the Digestive System (ARCAD) database led to similar outcomes. In the ARCAD study the probability of survival for patients with peritoneal metastases was significantly worse compared with single organ non-peritoneal metastases (adjusted HR $=0.73, p=$ 0.012 and $0.61, p<0.001$ for liver and lung metastases, respectively), but the difference was not significant when subgroup analysis performed between the patients with isolated peritoneal involvement and patients with metastases in two non-peritoneal organs (adjusted HR $=1.10$, $p=0.37) .^{22}$

In our study the survival was not affected by the type of adjuvant therapy regimen. The study of Colucci and co-workers showed no significant differences between FOLFOX4 and FOLFIRI combinations in terms of response rate and OS. ${ }^{23}$ Also the review of Pasetto and others that was designed to analyze FOLFOX versus FOLFIRI did not reach to a firm conclusion about the choice of treatment in metastatic CRC. ${ }^{24}$

In the current study addition of monoclonal antibodies to treatment of patients with metastatic CRC did not lead to significantly better overall survival. There are similar findings available in the literature. The study of Saltz and colleagues on 1401 patients with metastasis revealed that adding bevacizumab to an oxiliplatin based chemotherapy did not significantly affect $\mathrm{OS}(\mathrm{HR}=0.89$; 97.5\% CI: $0.76-1.03, p=0.077)$; although in that study HR of PFS reached the statistical level of significance $(\mathrm{HR}=0.63 ; 97.5 \% \mathrm{CI}: 0.52-0.75, p<0.001) .{ }^{9}$ Study of Guren and colleagues in NORDIC-VII patients advocated that adding cetuximab to Nordic FLOX regimen did not have any clinical advantage regarding OS and PFS in the first line treatment of metastatic CRC. ${ }^{10}$ The study of Van Cutsem and others showed that using cetuximab in addition to an irinotecan-based chemotherapy (FOLFIRI protocol) could reduce the risk or progression $(\mathrm{HR}=0.85$; 95\% CI: $0.72-0.99 ; p=0.048)$, but the effect on death was not significant (adjusted HR $=0.93 ; 95 \%$ CI: 0.81 $1.07 ; p=0.31) .{ }^{25}$

Enormous studies concerning the treatment of advanced CRC focused on molecular features. Several biomarkers were introduced. Among them, molecular testing for KRAS and NRAS tumor genes are recommended in advanced CRC. Almost half of the patients with advanced CRC harbor KRAS or NRAS mutations, which is a negative predictive factor for response to antiEGFR monoclonal antibodies. ${ }^{26}$

Treatment of advanced CRC in Iran and its survival results seems to be in line with centers in developed countries. Notwithstanding advances in the treatment of lymph node positive and metastatic CRC, it appears that clinical and pathological features might yet have more important roles in the prognosis of the patients.

\section{ACKNOWLEDGMENTS}

There is no funding from any institutions for this study. 


\section{ETHICAL APPROVAL}

There is nothing to be declared.

\section{CONFLICT OF INTEREST}

The authors declare no conflict of interest related to this work.

\section{REFERENCES}

1. Siegel RL, Miller KD, Jemal A. Cancer statistics, 2016. CA Cancer J Clin 2016;66:7-30. doi:10.3322/caac.21332.

2. Torre LA, Siegel RL, Ward EM, Jemal A. Global Cancer Incidence and Mortality Rates and Trends--An Update. Cancer Epidemiol Biomarkers Prev 2016;25:16-27. doi: 10.1158/1055-9965.EPI-15-0578.

3. Dolatkhah R, Somi MH, Kermani IA, Ghojazadeh M, Jafarabadi MA, Farassati F, et al. Increased colorectal cancer incidence in Iran: a systematic review and metaanalysis. BMC Public Health 2015;15:997. doi:0.1186/ s12889-015-2342-9.

4. Siegel R, Naishadham D, Jemal A. Cancer statistics, 2012. CA Cancer J Clin 2012;62:10-29. doi:10.3322/caac.20138.

5. Taal BG, Van Tinteren H, Zoetmulder FA, NACCP group. Adjuvant 5FU plus levamisole in colonic or rectal cancer: improved survival in stage II and III. $\mathrm{Br} J$ Cancer 2001;85:1437-43. doi:10.1054/bjoc.2001.2117.

6. De Gramont A, Krulik M, Cady J, Lagadec B, Maisani JE, Loiseau JP, et al. High-dose folinic acid and 5-fluorouracil bolus and continuous infusion in advanced colorectal cancer. Eur J Cancer Clin Oncol 1988;24:1499-503. doi: 10.1016/0277-5379(88)90341-0.

7. Saltz LB, Cox JV, Blanke C, Rosen LS, Fehrenbacher $\mathrm{L}$, Moore MJ, et al. Irinotecan plus fluorouracil and leucovorin for metastatic colorectal cancer. Irinotecan Study Group. N Engl J Med 2000;343:905-14. doi:10.1056/ NEJM200009283431302.

8. Goldberg RM, Sargent DJ, Morton RF, Fuchs CS, Ramanathan RK, Williamson SK, et al. A randomized controlled trial of fluorouracil plus leucovorin, irinotecan, and oxaliplatin combinations in patients with previously untreated metastatic colorectal cancer. J Clin Oncol 2004;22:23-30. doi:10.1200/JCO.2004.09.046.

9. Saltz LB, Clarke S, Diaz-Rubio E, Scheithauer W, Figer $\mathrm{A}$, Wong $\mathrm{R}$, et al. Bevacizumab in combination with oxaliplatin-based chemotherapy as first-line therapy in metastatic colorectal cancer: a randomized phase III study. J Clin Oncol 2008;26:2013-9. doi:10.1200/ JCO.2007.14.9930.

10. Guren TK, Thomsen M, Kure EH, Sorbye H, Glimelius B, Pfeiffer P, et al. Cetuximab in treatment of metastatic colorectal cancer: final survival analyses and extended RAS data from the NORDIC-VII study. Br $J$ Cancer 2017;116:1271-8. doi:10.1038/bjc.2017.93.

11. Glimelius B, Dahl O, Cedermark B, Jakobsen A, Bent- zen SM, Starkhammar H, et al. Adjuvant chemotherapy in colorectal cancer: a joint analysis of randomised trials by the Nordic Gastrointestinal Tumour Adjuvant Therapy Group. Acta Oncologica 2005;44:904-12. doi: 10.1080/02841860500355900a.

12. de Gramont A, Figer A, Seymour M, Homerin M, Hmissi A, Cassidy J, et al. Leucovorin and fluorouracil with or without oxaliplatin as first-line treatment in advanced colorectal cancer. J Clin Oncol 2000;18:2938-47. doi:10.1200/JCO.2000.18.16.2938.

13. Laurie JA, Moertel CG, Fleming TR, Wieand HS, Leigh JE, Rubin J, et al. Surgical adjuvant therapy of large-bowel carcinoma: an evaluation of levamisole and the combination of levamisole and fluorouracil. The North Central Cancer Treatment Group and the Mayo Clinic. J Clin Oncol 1989;7:1447-56. doi:10.1200/JCO.1989.7.10.1447.

14. Yothers G, O’Connell MJ, Allegra CJ, Kuebler JP, Colangelo LH, Petrelli NJ, et al. Oxaliplatin as adjuvant therapy for colon cancer: updated results of NSABP C-07 trial, including survival and subset analyses. J Clin Oncol 2011;29:3768-74. doi:10.1200/JCO.2011.36.4539.

15. Andre T, Boni C, Navarro M, Tabernero J, Hickish T, Topham C, et al. Improved overall survival with oxaliplatin, fluorouracil, and leucovorin as adjuvant treatment in stage II or III colon cancer in the MOSAIC trial. J Clin Oncol 2009;27:3109-16. doi:10.1200/JCO.2008.20.6771.

16. Andre T, Boni C, Mounedji-Boudiaf L, Navarro M, Tabernero J, Hickish T, et al. Oxaliplatin, fluorouracil, and leucovorin as adjuvant treatment for colon cancer. $N E n g l$ J Med 2004;350:2343-51. doi:10.1056/NEJMoa032709.

17. Diaz-Rubio E, Gomez-Espana A, Massuti B, Sastre J, Abad A, Valladares M, et al. First-line XELOX plus bevacizumab followed by XELOX plus bevacizumab or single-agent bevacizumab as maintenance therapy in patients with metastatic colorectal cancer: the phase III MACRO TTD study. Oncologist 2012;17:15-25. doi:10.1634/theoncologist.2011-0249.

18. Adams RA, Meade AM, Seymour MT, Wilson RH, Madi A, Fisher D, et al. Intermittent versus continuous oxaliplatin and fluoropyrimidine combination chemotherapy for first-line treatment of advanced colorectal cancer: results of the randomised phase $3 \mathrm{MRC}$ COIN trial. Lancet Oncol 2011;12:642-53. doi:10.1016/S1470-2045(11)70102-4.

19. Tournigand C, Cervantes A, Figer A, Lledo G, Flesch M, Buyse M, et al. OPTIMOX1: a randomized study of FOLFOX4 or FOLFOX7 with oxaliplatin in a stopand-Go fashion in advanced colorectal cancer--a GERCOR study. J Clin Oncol 2006;24:394-400. doi:10.1200/ JCO.2005.03.0106.

20. Cassidy J, Clarke S, Diaz-Rubio E, Scheithauer W, Figer A, Wong R, et al. XELOX vs FOLFOX-4 as first-line therapy for metastatic colorectal cancer: NO16966 updated results. Br J Cancer 2011;105:58-64. doi:10.1038/ bjc.2011.201.

21. Gill S, Loprinzi CL, Sargent DJ, Thome SD, Alberts SR, Haller DG, et al. Pooled analysis of fluorouracil-based adjuvant therapy for stage II and III colon cancer: who 
benefits and by how much? J Clin Oncol 2004;22:1797806. doi:10.1200/JCO.2004.09.059.

22. Franko J, Shi Q, Meyers JP, Maughan TS, Adams RA, Seymour MT, et al. Prognosis of patients with peritoneal metastatic colorectal cancer given systemic therapy: an analysis of individual patient data from prospective randomised trials from the Analysis and Research in Cancers of the Digestive System (ARCAD) database. Lancet Oncol 2016;17:1709-19. doi:10.1016/S14702045(16)30500-9.

23. Colucci G, Gebbia V, Paoletti G, Giuliani F, Caruso M, Gebbia N, et al. Phase III randomized trial of FOLFIRI versus FOLFOX4 in the treatment of advanced colorectal cancer: a multicenter study of the Gruppo Oncologico Dell'Italia Meridionale. J Clin Oncol 2005;23:4866-75. doi:10.1200/JCO.2005.07.113.

24. Pasetto LM, Jirillo A, Iadicicco G, Rossi E, Paris MK, Monfardini S. FOLFOX versus FOLFIRI: a comparison of regimens in the treatment of colorectal cancer metastases. Anticancer Res 2005;25:563-76.

25. Van Cutsem E, Kohne CH, Hitre E, Zaluski J, Chang Chien CR, Makhson A, et al. Cetuximab and chemotherapy as initial treatment for metastatic colorectal cancer. N Engl J Med 2009;360:1408-17. doi:10.1056/NEJMoa0805019.

26. Lievre A, Bachet JB, Le Corre D, Boige V, Landi B, Emile $\mathrm{JF}$, et al. KRAS mutation status is predictive of response to cetuximab therapy in colorectal cancer. Cancer Res 2006;66:3992-5. doi:10.1158/0008-5472.CAN-06-0191. 